\title{
Role of microsurgical varicocelectomy in the management of non-obstructive azoospermia with varicocele: our tertiary care centre experience
}

\author{
Avinash P. S. Thakur ${ }^{1 *}$ (D) Darsan Sadasivan², Vivek Sharma ${ }^{3}$, Vasantharaja Ramasamy², Subeesh Parol ${ }^{2}$, \\ Sangeeta Singh ${ }^{4}$ and Jatin Soni ${ }^{2}$
}

\begin{abstract}
Background: Varicocele is an abnormal dilation and tortuosity of the internal spermatic veins within the pampiniform plexus of the spermatic cord. Varicocele is associated with progressive testicular damage and infertility. Azoospermia is associated with a varicocele in approximately $4-14 \%$ cases. For men with azoospermia or severe oligoasthenospermia, varicocele repair may result in modest improvement in semen quality which may have a significant advantage on couple's fertility options. The aim of the study was to evaluate the role of microsurgical varicocelectomy in the men of non-obstructive azoospermia (NOA) with clinical varicocele.

Methods: This was a retrospective study conducted between August 2012 and January 2017, a backward review of 104 patients with the diagnosis of infertility and NOA with palpable varicocele that underwent microsurgical varicocelectomy at our institution was performed. In addition, microdissection testicular sperm extraction (MDTESE) results of these post-varicoceletomy patients were compared with the patients of NOA without varicocele.

Results: A total of 104 patients underwent varicocelectomy; out of these, 19 patients (18.26\%) had sperm on sperm analysis post-operatively. Two of them had spontaneous pregnancy (10.5\%), and 3 had children by intracytoplasmic sperm injection (15.78\%). Out of the 85 patients who had MDTESE, 29 patients $(34.11 \%)$ had sperms in their testis. The fertilization rate was $89.65 \%$. Sperm retrieval rate (SRR) in NOA men with varicocele was $34.11 \%$ which was higher from those who had NOA without varicocele (24.03\%). Live birth rate was 31.03\% in NOA men who had varicocelectomy which was more in comparison to NOA men without varicocele (24\%).

Conclusions: In NOA men with varicocele microsurgical varicocelectomy may have favourable effects which results in recovery of motile sperms in the post-operative ejaculate and also on spontaneous or assisted pregnancies, but it appears that this effect was more remarkable on MDTESE results when following successful intracytoplasmic sperm injection. Importantly, Sperm retrieval rate, pregnancy rate and subsequent live birth rate were higher in these patients in comparison to patients affected by NOA alone. In patients with NOA and coexisting varicocele, varicocelectomy can be considered to be essential to the overall reproductive outcome in these patients.
\end{abstract}

Keywords: Azoospermia, Microscopic varicocelectomy, Varicocele, Infertility

\footnotetext{
*Correspondence: drapst@gmail.com

1 Department of Urology, Super Speciality Hospital, Netaji Subhash

Chandra Bose Medical College, Jabalpur, M.P., India

Full list of author information is available at the end of the article
} 


\section{Background}

Varicocele is an abnormal dilation and tortuosity of the internal spermatic veins within the pampiniform plexus of the spermatic cord. Varicocele is associated with progressive testicular damage and infertility. It is also related with bilateral spermatogenic abnormalities and Leydig cell dysfunction in infertile men.

Increased scrotal temperature due to impaired drainage of blood in the pampiniform plexus of the spermatic cord by varicocele is believed to cause spermatogenesis deterioration progressively [1]. Varicocele is a common problem in cases of infertility, and it is seen in approximately $10-20 \%$ of the normal male population, in $35-40 \%$ of the men with primary infertility and between 75 and $80 \%$ of men with secondary infertility [2-4]. Varicocele can result in generalized impairment of sperm parameters characterized by abnormal semen quality, varying from oligospermia to complete azoospermia $[1,5]$. The finding of azoospermia or severe oligospermia with varicocele is reported to range from 4.3 to $13.3 \%$ [6].

Varicocelectomy improves the spermatogenesis and also the function of Leydig cells [4]. Although it has been well reported that sperm parameters have been improved in oligoasthenoteratozoospermia (OAT) patients after varicocelectomy, its value is still not defined in men with azoospermia [7]. There is a clinical evidence to suggest that focal or 'patches' of sperm production occurs in damaged testes. It is assumed that in men with nonobstructive azoospermia (NOA), testis might show a homogeneously or a randomly distributed spermatogenesis. In the first condition, a large part of testicular tissue represent the spermatogenesis, whereas in the latter condition a large part of testicular tissue might be devoid of focal advanced spermatogenesis [8].

Azoospermia is associated with a varicocele in approximately $4-14 \%$ cases $[6,9]$. In previous studies, the outcome of varicocelectomy in these men was shown to be less significant [7], but as per recent studies in men with azoospermia there is modest improvement in semen parameters after varicocele repair $[7,9,10]$.

The primary advantage of varicocelectomy in cases of NOA is the possibility of getting motile sperms in the ejaculate. Other benefit of varicocele repairs in azoospermic men is that by producing motile sperms from the fresh ejaculate there are increased success rates of assisted reproductive techniques (ART) such as intracytoplasmic sperm injection (ICSI) or testicular sperm extraction (TESE) $[9,11,12]$.

According to some studies in patients with azoospermia, there is about $40 \%$ improvement of semen parameters and also in the production of motile sperms in the ejaculate after varicocele repair using microsurgical technique $[13,14]$. For men with azoospermia or severe oligoasthenospermia, varicocele repair may result in modest improvement in semen quality which may have a significant advantage on couple's fertility options [11].

Thus, the aims of our study were to evaluate the role of microsurgical subinguinal varicocelectomy in men of NOA with varicocele, to determine the predictive parameters of post-operative improvement and to assess the net result of varicocelectomy on sperm retrieval rate, pregnancy rate and birth rate.

\section{Methods}

This was a retrospective observational cohort study, conducted between August 2012 and January 2017; a total of 104 patients with the diagnosis of infertility and NOA with clinical varicocele underwent microsurgical varicocelectomy at our institution. A detailed informed consent was obtained from all patients before the surgical procedure.

\subsection{Inclusion criteria}

All infertile patients with NOA and palpable varicocele on their physical examination were included.

\subsection{Exclusion criteria}

1 Non-palpable varicocele.

2 Female factor infertility.

3 Obstructive azoospermia.

4 Genetic abnormalities like Y-chromosome microdeletion and Klinefelter syndrome.

Infertility is defined as failure to achieve a pregnancy after 12 months or more with unprotected intercourse.

The cases of varicocele were detected by scrotal examinations, with the patients in standing position and also during valsalva's manoeuvre. To confirm the presence of varicocele and to evaluate testicular size scrotal, colour Doppler ultrasound was used. The varicocele was categorized into three grades: grade 1 when it was palpable just during the valsalva's manoeuvre, grade 2 when it was palpable without the manoeuvre, and grade 3 when it was visible. In all patients at least 2 semen analyses were obtained by masturbation after 3-5 days of abstinence. These two semen analyses were separated by a minimum interval of 2 weeks. Semen samples were collected and evaluated according to WHO criteria.

All patient records were also reviewed for demographical, clinical, diagnostic and procedural data. Patients age, infertility duration, testis volume, serum testosterone (T), follicle-stimulating hormone (FSH), luteinizing hormone (LH), testicular ultrasound findings, testicular biopsy results, genetic abnormalities, post-operative complications, sperms in ejaculated semen, sperm retrieval rate in 
microdissection testicular sperm extraction (MDTESE), fertilization rate, pregnancy and birth rate were analysed.

\subsection{Surgical technique}

In this study, varicocele repair was performed using a microsurgical subinguinal approach with lymphatic and artery sparing technique. Initial step was small transverse skin incision in the area of external inguinal ring; the spermatic cord was then grasped with a Babcock clamp and delivered through the incision. The external spermatic perforators and gubernacular veins were isolated and divided. The cord was examined under the magnification of an operating microscope. After the external and internal fascias were incised, the underlying external and internal spermatic arteries were detected by their subtle pulsations. After dissecting from the underlying veins these arteries were encircled with a 3-0 silk suture just for identification. Lymphatics were preserved to prevent the development of post-operative lymphocele and hydrocele. With the exception of the vasal veins, all internal spermatic veins ligated and divided. In the last haemostasis achieved and incision closed.

At the same time of the varicocele repair, every patient underwent diagnostic testicular core biopsy. Based on size and consistency of testis, biopsies were taken from the healthier appearing testis. These biopsies were analysed and classified by an experienced pathologist as maturation arrest, sertoli-cell-only (SCO) pattern, hypospermatogenesis and normal spermatogenesis.

After the varicocele repair, semen analyses were obtained at 3, 6 and 12 (if required) months in post-operative evaluation. For data analysis, most improved postoperative semen analysis was used.

In order to avoid retrieval of testicular sperms, all NOA with varicocele patients who underwent microsurgical varicocelectomy were checked to find out whether they had enough sperms in ejaculate post-operatively. By using post-operative ejaculated sperms, the assisted and unassisted pregnancy rates were evaluated.

In addition, MDTESE results analysed in these patients (in whom post varicocelectomy semen was negative for sperms) and their fertilization, conception and delivery rates also reviewed.

In $5(4.7 \%)$ patients hydrocele formation (post-operatively) occurred; out of these two patients were managed by aspiration and other 3 by surgical repair.

In last, these 104 patients' results were compared to other 104 patients of NOA without evidence of any varicocele. The variables of semen parameter changes, hormonal levels, sperm retrieval rate and pregnancy achievement rate were correlated with different clinical, laboratory and pathological parameters. All patients in this study had been treated in Government Medical Institute. Patient information for this study remained confidential.

\subsection{Statistical analysis}

Data values were entered according to the variables onto spread sheets of Microsoft Office Excel, and the variables were analysed using standard analytical techniques. The associations between study variables were analysed using Chi-square test and Student's $t$ test. ' $p$ ' values $<0.05$ were considered significant. For the variables which were categorical, percentage and proportions were calculated manually.

\section{Results}

From August 2012 to January 2017, a total of 104 patients were enrolled in this study. The mean age of the patients was $31.8 \pm 3.6$ years (range 26-42), and the mean infertility duration was $2.9 \pm 1.2$ years (range $1-7$ ). The total testicular volume mean value (i.e. both testis volume in each patient) was $17.70 \pm 5.9 \mathrm{cc}$.

These patients treated for varicocelectomy had analysis of karyotype and azoospermia factor (AZF) microdeletion; none of the patient had microdeletion, and in all patients karyotype was $46 \mathrm{XY}$.

Out of 104 patients, $8(7.6 \%)$ had grade I varicocele, 36 patients (34.6\%) had grade II varicocele while 60 patients (57.6\%) had grade III varicocele. Bilateral varicocelectomy was done in 72 patients, while 26 and 6 patients had unilateral left and right varicocelectomy performed respectively.

In post-operative sperm analysis of these 104 patients, 19 patients $(18.26 \%)$ acquired motile sperms, with the mean follow-up time of 4.4 months (range 3-12). After varicocelectomy, ejaculate was positive for sperms in 13 of 60 patients (21.6\%) with grade III, 6 of 36 patients $(16.6 \%)$ with grade II and none of 8 patients with grade I varicocele, this finding indicates that the higher grade of varicocele might be associated with better results postoperatively. In biopsy reports of these 19 patients, hypospermatogenesis had seen in 14, maturation arrest in 3 , and sertoli-only syndrome (SOS) in 2 patients. In these patients, the mean sperm concentration was $1.39 \pm 0.05 \times 10^{6} / \mathrm{mL}$ and mean total sperm motility was $39.04 \pm 3.9 \%$ (Table 1 ).

The improvement in sperm concentration and motility was greater in patients of bilateral varicocelectomy than unilateral varicocelectomy. Out of the 19 patients who acquired motile sperms after varicocelectomy, successful pregnancy occurred in 5 patients (couples). All these 5 patients had bilateral varicocelectomy for bilateral grade III varicocele in 4 patients and bilateral grade II varicocele in 1 patient. In these 5 , two patients 
Table 1 Preoperative characteristics and post-operative outcome of varicocele patients

\begin{tabular}{|c|c|c|c|}
\hline Varicocele & & Cases & Control \\
\hline \multirow[t]{3}{*}{ Grade } & Grade 1 & 08 & 0 \\
\hline & Grade 2 & 36 & 0 \\
\hline & Grade 3 & 60 & 0 \\
\hline \multirow[t]{3}{*}{ Location } & Left & 26 & 0 \\
\hline & Right & 06 & 0 \\
\hline & Bilateral & 72 & 0 \\
\hline \multirow[t]{3}{*}{ Testis condition } & Normal testis & 30 & 42 \\
\hline & Unilateral atrophic & 18 & 04 \\
\hline & Bilateral atrophic & 56 & 58 \\
\hline \multirow[t]{4}{*}{ Presence of sperm after varicocelectomy } & & 19/104 & NA \\
\hline & In grade 1 & $0 / 8$ & \\
\hline & In grade 2 & $6 / 36$ & \\
\hline & In grade 3 & $13 / 60$ & \\
\hline Mean sperm concentration $\left(\times 10^{6} / \mathrm{mL}\right)$ & & $1.39 \pm 0.05$ & NA \\
\hline Mean sperm motility (\%) & & $39.04 \pm 3.9$ & NA \\
\hline Pregnancy (total) & & 5 & \\
\hline
\end{tabular}

had spontaneous pregnancy $(2 / 19=10.5 \%)$ and other 3 had children through intracytoplasmic sperm injection $(3 / 19=15.78 \%)$.

In the remaining 85 patients $(81.7 \%)$, who had negative sperms post-operatively, microdissection testicular sperm extraction (MDTESE) was done. Between varicocelectomy and micro-TESE, the mean interval was $9.0 \pm 2.7$ months (range 6-18). In these patients, before the varicocelectomy, grades I, II and III of varicocele were in 8 (9.4\%), $30(35.2 \%)$ and 47 (55.2\%) patients, respectively.

In these 85 patients who had MDTESE, 29 patients (34.11\%) had sperms in their testis. In the biopsy reports of these 29 patients, hypospermatogenesis had reported in 18, maturation arrest in 6, and sertoli-only syndrome (SOS) in 5 patients. In addition, of these 29 patients three had no egg fertilization; therefore, the fertilization rate was 26/29 (89.65\%) (Table 2).
Characteristics of the case group are compared to other 104 patients with NOA without varicocele (control group). For the case and control, patients mean age was $31.8 \pm 3.6$ years and $31.4 \pm 3.3$ years, respectively $(p=0.43)$. FSH level was $16.02 \pm 6.2 \mathrm{mIU} / \mathrm{mL}$ in the control group and $14.8 \pm 5.4 \mathrm{mIU} / \mathrm{mL}$ in the case group which was lower but not significantly $(p=0.13)$. No significant difference was observed in the other clinical or laboratory parameters between these two groups (Table 3).

After MDTESE in the case and the control groups, sperm retrieval rate was 29/85 (34.11\%) and 25/104 (24.03\%), respectively $(\mathrm{OR}=1.63,95 \%$ CI $0.86-3.08$, $p=0.127)$. The pregnancy and live birth rate in the case and the control groups were $31.03 \%$ ( 9 of 29 cases) and $24 \%$ (6 of 25 controls), respectively (OR $=1.42,95 \% \mathrm{CI}$ $0.42-4.77, p=0.565$ ) (Table 2).

Table 2 Post-MDTESE results with comparison

\begin{tabular}{llll}
\hline Variable & Cases $(\boldsymbol{N = 1 0 4 )}$ & Control $(\boldsymbol{N = 1 0 4 )}$ & $\boldsymbol{p}$ value \\
\hline Sperm retrieval rate [SRR] (after MDTESE) & $29 / 85(34.11 \%)$ & $25 / 104(24.03 \%)$ & \\
Testicular histopathology (in sperm retrieval positive patients) & & \\
Hypospermatogenesis & $18 / 29$ & $16 / 25$ \\
Maturation arrest & $06 / 29$ & $05 / 25$ \\
Sertoli-only-cell (SCO) pattern & $05 / 29$ & $04 / 25$ \\
Fertilization rate & $26 / 29(89.6 \%)$ & $21 / 25(84 \%)$ \\
Pregnancy rate & $9 / 29(31.03 \%)$ & $6 / 25(24 \%)$ & 0.537 \\
Live birth rate & $9 / 29(31.03 \%)$ & $6 / 25(24 \%)$ & 0.565 \\
\hline
\end{tabular}


Table 3 Characteristics of the patients

\begin{tabular}{llll}
\hline Variable & Cases $(\boldsymbol{N}=\mathbf{1 0 4})$ & Control $(\boldsymbol{N}=\mathbf{1 0 4})$ & $\boldsymbol{p}$ value \\
\hline Age in years (mean $\pm \mathrm{SD}$, range) & $31.8 \pm 3.6(26-42)$ & $31.4 \pm 3.3(26-41)$ & 0.43 \\
Duration of infertility in year (mean, range) & $2.9+1.2(1-7)$ & $2.7+1.4(1-6)$ & 0.51 \\
Hormones level & & & \\
FSH $(\mathrm{mlU} / \mathrm{mL})$ & $14.8+5.4$ & $16.02+6.2$ & 0.13 \\
$\mathrm{LH}(\mathrm{mlU} / \mathrm{mL})$ & $7.5+2.7$ & $7.6+2.5$ & 0.72 \\
Testosterone $(\mathrm{ng} / \mathrm{mL})$ & $4.3+2.0$ & $4.6+1.8$ & 0.19 \\
Mean testicular volume $\left(\mathrm{cm}^{3}\right)$ & $17.7 \pm 5.9$ & $18.12 \pm 5.6$ & 0.60 \\
\hline
\end{tabular}

SD Standard deviation

' $p$ ' values calculated using Student's $t$ test for quantitative variables and Chi-square test for qualitative variables-values less than 0.05 were taken as significant

\section{Discussion}

Although the beneficial effects of varicocelectomy have been well studied in oligoasthenoteratozoospermia (OAT) men, the benefits of this surgical procedure are still limited and controversial in men of non-obstructive azoospermia $[15,16]$. In 1952, the first study on the importance of varicocelectomy for treatment of NOA was performed by Tulloch [17]; after that, several studies investigated the effects of varicocelectomy on NOA patients $[7,9,10,11,13,14]$.

Testicular blood drains by pampiniform plexus, and when dilatation of these plexus vessels happens, varicocele occurs. The small valves normally prevent the reverse blood flow, but when there is compression of the veins or defects in these valves develops vessel dilatation occurs. Through several mechanisms, varicocele can cause generalized deterioration and progressive impairment of sperm production and result in abnormal semen quality, varying from oligospermia to complete azoospermia $[2,5]$. The disease can change the concentration, motility, morphology and structure of sperm DNA $[18,19]$.

The complete absence of sperm in the ejaculate is defined as azoospermia. It is found in up to $15 \%$ of infertile men [18-20]. For the proper management of patients in azoospermia condition, it is important to differentiate between obstructive and non-obstructive pathology [18-21]. Testicular function can be demonstrated by FSH, LH, total testosterone and oestradiol levels, but measurement of fructose level in semen helps to rule out obstructive diagnosis [18-22].

On karyotype analysis, Y-chromosome microdeletions related to around $15 \%$ of patients with NOA, while Klinefelter syndrome is one of the most frequent genetic alterations in azoospermic men [18, 23, 24]. The Y-chromosome has three regions known as AZFa, $\mathrm{AZFb}$ and AZFc, and they incorporate the information for the production and maturation of sperm cells [24]. Evaluation of this is really essential for counselling because these patients can transmit genetic disorders to the offspring and they are not likely to benefit from varicocele surgery [19, 21, 22, 24].

Azoospermia is an extreme testicular dysfunction in varicocele men and is a significant barrier to unassisted pregnancy. In recent literature, there are several studies focussed on the association between varicocele and azoospermia. In this study, we also investigated the relationship between these two and evaluated the effects of microsurgical varicocelectomy on azoospermia men.

Varicocele repair by the affected spermatic veins occlusion can result in improvements in semen parameters, scrotal discomfort and spontaneous pregnancy rates [25]. Surgical repair can be performed by inguinal, retroperitoneal, subinguinal or laparoscopic approaches [26].

For men with NOA who desires pregnancy contribution by their own biological materials, treatment options include TESA (testicular sperm aspiration) or TESE with ICSI. Our study suggested that in men who have NOA with varicocele, the options of reproduction increases following varicocelectomy. In NOA men, the real advantage of this procedure is the possibility of producing motile sperms in the ejaculate. The use of motile sperm from fresh ejaculate is preferable to TESE in preparation for ICSI [27]. Compared with sperm retrieved by TESE, the fresh ejaculated sperms have superior ICSI success rates. Another advantage is to avoid the invasive and potentially damaging procedure of TESE [28].

In this study, we treated a total of 104 patients of NOA with clinical varicocele by microsurgical subinguinal approach. After varicocele repair in these 104 patients, we detected motile sperms in the ejaculate of 19 patients (18.26\%) and spontaneous pregnancy occurred in $2 / 19$ (10.5\%) patients. However, assisted reproductive technique was required in the majority of couples to initiate pregnancy.

The results of our study and the previous studies of microsurgical varicocelectomy are compared. 
Matthews et al. [11] reported 54.5\% (12 out of 22) patients had motile sperm in the ejaculate and $27.7 \%$ spontaneous pregnancy rate after subinguinal microsurgical varicocelectomy. The study by Schlegel et al. [7] shows that $22.5 \%$ (7 of 31) patients had motile sperm in the ejaculate and $3.2 \%$ spontaneous pregnancy rate after varicocelectomy. Several studies on NOA have shown improvement benefits in semen parameters of $20-50 \%$ cases and even in spontaneous pregnancy rate [9, 13, 29]. Kim et al. [30] on the other side reported that despite a mean follow-up of 24 months, spontaneous pregnancy was not seen even a single patient of complete azoospermia ( 0 of 28 ).

Another finding of our study was that, in men of NOA with varicocele, treatment response (presence of sperms in ejaculate after varicocelectomy) was better in patients with higher grade and bilateral varicocele. A study by Kadioglu et al. [9] also reported the similar finding.

Testicular histology is considered to be a significant predictive factor of outcome $[9,11,30]$. Patients with maturation arrest at the spermatocyte stage and germ cell aplasia had not shown improved semen quality; on the other side, azoospermic men with hypospermatogenesis or maturation arrest at the spermatid stage had shown improved semen quality post-operatively $[5,11$, 14]. In this study, testicular biopsy of 19 patients who had achieved motile sperm post-varicocelectomy we found hypospermatogenesis in 14 patients. So, this result again supports that the most important histopathological predictor of post-operative sperm in the ejaculate was hypospermatogenesis.

It is important to note that among the 104 patients, only 2 patients (1.9\%) with SCO pattern were positive for motile sperms in the post-surgery semen analyses which occurred at 6 and 8 months post-operatively. On further follow-up, these patients relapsed again into azoospermia. This finding suggests that varicocele repair may induce spermatogenesis temporarily which could not be sustained for a long time. So in this subgroup of patients, once the patient has sperms in the semen, semen cryopreservation should be considered. Testicular biopsy data of this study suggest that some level of sperm production is essential for a good result after varicocelectomy. Few sperm production region may present in patients of SCO [31].

Our data did not show any relation between presence of unilateral or bilateral testicular atrophy and final result. These findings are near to those reported in a previous study [11].

Previous studies reported post-varicocelectomy hydrocele incidence around $6-10 \%$ of patients $[32,33]$. In our study, hydrocele formation occurred in $4.8 \%(5 / 104)$ patients. Improvement in semen quality and fertility were not significantly affected by post-operative hydrocele formation, this is similar to findings from previous studies [34].

The clinical advantage of varicocele surgery is that a considerable number of men with NOA have an option to provide sperms by ejaculation, without the need of invasive testicular sperm retrieval procedures.

Pregnancy can be achieved even with only a single sperm due to advancement in assisted reproductive technology (ART). Small improvements in spermatogenesis may exert a profound effect on couples' reproductive options. Recent reports of TESE in men with NOA indicate that sperms are recovered from testicular tissue in $50-60 \%$ of such men $[35,36]$.

In this study, SRR by MDTESE was $34.11 \%$ (29/85) in NOA men with varicocelectomy compared to $24.03 \%$ $(25 / 104)$ in NOA cases without varicocele. The study by Inci et al. [23] showed the SRR 53\% and 30\% in treated and untreated group, respectively. Therefore, Sperm retrieval rate may increase after varicocele surgery in NOA men with varicocele as compared to NOA men with no varicocele. Live birth rate/embryo was $31.03 \%$ $(9 / 29)$ in NOA men with varicocele in comparison with $24 \%(6 / 25)$ in NOA without varicocele.

These results suggest that microsurgical varicocelectomy should be considered in all patients of NOA with clinical varicocele before proceeding directly to TESE.

The present study was nonrandomized, retrospective with small sample size which limits the generalization of these findings. To validate these results, further prospective studies with randomization and large sample sizes are required in future.

\section{Conclusion}

According to this study, we propose that in NOA with clinical varicocele patients, microsurgical varicocelectomy may have favourable effects which results in recovery of motile sperms in the post-operative ejaculate and also on spontaneous or assisted pregnancies, but it appears that this effect is more remarkable on MDTESE results when following successful ICSI. Importantly, sperm retrieval rate, pregnancy rate and subsequent live birth rate were higher in these patients in comparison to patients affected by NOA alone. The common histopathological findings in MDTESE-positive patients were hypospermatogenesis and maturation arrest that indicates the need of MDTESE for sperm retrieval in such patients.

So, in patients of NOA with varicocele, varicocele surgery can be considered an essential factor to improve the SRR in micro-TESE. Occasionally, it offers a viable alternative to TESE and ICSI or donor insemination and result in unassisted pregnancy. Nevertheless, the majority of 
infertile couple with NOA men should be counselled that ART will most likely be required to initiate a pregnancy.

Although results of our study are very promising, further well-designed, randomized studies with a larger population and a longer follow-up period are needed to confirm its beneficial effect.

\section{Abbreviations}

OAT: Oligoasthenoteratozoospermia; NOA: non-obstructive azoospermia; ART : assisted reproductive techniques; ICSI: intracytoplasmic sperm injection; TESE: testicular sperm extraction; MDTESE: microdissection testicular sperm extraction; FSH: follicle-stimulating hormone; LH: luteinizing hormone; SCO: sertoli-cell-only; AZF: azoospermia factor; SRR: sperm retrieval rate.

\section{Acknowledgements}

We acknowledge the contribution of entire research team including pathology and gynaecology departments. We also acknowledge all the patients who participated in the study.

\section{Authors' contributions}

APST prepared the manuscript, analysed the data and supervised the study; DS helped with data collection and designed the study; VS and VR helped in critical revision of manuscript for important intellectual contents; SP and SS helped in manuscript preparation and drafting of manuscript; and APST, DS and JS performed literature search. All authors read and approved the final manuscript.

\section{Funding}

Not applicable.

\section{Availability of data and materials}

The datasets used and analysed during the current study is available from the corresponding author on reasonable request.

\section{Ethics approval and consent to participate}

The ethics committees name is 'Human Ethics Committee', and date of approval was 24/03/2017. Informed written consent to participate in the study was obtained from all the patients.

\section{Consent for publication}

Not applicable.

\section{Competing interests}

The authors declare that they have no competing interests.

\section{Author details}

${ }^{1}$ Department of Urology, Super Speciality Hospital, Netaji Subhash Chandra Bose Medical College, Jabalpur, M.P., India. ${ }^{2}$ Department of Urology, Super Speciality Block, Government Medical College, Thiruvananthapuram, Kerala, India. ${ }^{3}$ Department of Urology, Super Speciality Hospital, Shyam Shah Medical College, Rewa, M.P., India. ${ }^{4}$ Triveni Institute of Dental Sciences Hospital and Research Centre, Bilaspur, C.G., India.

Received: 7 May 2020 Accepted: 2 September 2020 Published online: 03 November 2020

\section{References}

1. Dohle GR, Colpi GM, Hargreave TB, Papp GK, Jungwirth A, Weidner W (2005) EAU working group on male infertility EAU guidelines on male infertility. Eur Urol 48:703-711

2. World Health Organization (1992) The influence of varicocele on parameters of fertility in a large group of men presenting to infertility clinics. Fertil Steril 57:1289-1293

3. Stephen EH, Chandra A (2006) Declining estimates of infertility in the United States: 1982-2002. Fertil Steril 86:516-523
4. Wein AJ, Kavoussi LR, Partin AW, Peters CA (2015) Campbell Walsh urology, 11 th edn. Elsevier, Philadeiphia, p 604

5. Gorelick Jl, Goldstein M (1993) Loss of fertility in men with varicocele. Fertil Steril 59:613-616

6. Czaplicki M, Bablok L, Janczewski Z (1979) Varicocelectomy in patients with azoospermia. Arch Androl 3:51-55

7. Schlegel PN, Kaufmann J (2004) Role of varicocelectomy in men with nonobstructive azoospermia. J Fertil Steril 81(6):1585-1588

8. Turek PJ, Cha I, Ljung BM (1997) Systemic fine-needle aspiration of the testis: correlation to biopsy and results of organ "mapping" for mature sperm in azoospermic men. Urology 49:743-748

9. Kadioglu A, Tefekli A, Cayan S, Kandirali E, Erdemir F, Tellaloglu S (2001) Microsurgical inguinal varicocele repair in azoospermic men. Urology 57:328-333

10. Cakan M, Altug U (2004) Induction of spermatogenesis by inguinal varicocele repair in azoospermic men. Arch Androl 50:145-150

11. Matthews GJ, Matthews ED, Goldstein M (1998) Induction of spermatogenesis and achievement of pregnancy after microsurgical varicocelectomy in men with azoospermia and severe oligoasthenospermia. Fertil Steril 70:71-75

12. Su LM, Palermo GD, Goldstein M, Veeck LL, Rosenwaks Z, Schlegel PN (1999) Testicular sperm extraction with intracytoplasmic sperm injection for nonobstructive azoospermia: testicular histology can predict success of sperm retrieval. J Urol 161:112-116

13. Youssef T, Abd-Elaal E, Gaballah G, Elhanbly S, Eldosoky E (2009) Varicocelectomy in men with nonobstructive azoospermia: is it beneficial? Int J Surg 7:356-360

14. Weedin JW, Khera M, Lipshultz LI (2010) Varicocele repair in patients with nonobstructive azoospermia: a meta-analysis. J Urol 183:z2309-z2315

15. Witt MA, Lipshultz LI (1993) Varicocele: a progressive or static lesion? Urology 42:541-543

16. Kibar Y, Seckin B, Erduran D (2002) The effects of subinguinal varicocelectomy on Kruger morphology and semen parameters. J Urol 168:1071-1074

17. Tulloch WS (1951-1952) A consideration of sterility factors in the light of subsequent pregnancies.II.Sub fertility in the male.(Tr.Edinburgh Obst.Soc.Session 104). Edinb Med J 59:29-34

18. Mulhall JP, Stalh PJ, Varicocele SD (2013) Clinical cares pathways in andrology. Springer, Philadelphia, pp 165-170

19. Male Infertility Best Practice Policy Committee of the American Urological Association; Practice Committee of the American Society for Reproductive Medicine (2004) Report on varicocele and infertility. Fertil Steril 85(Suppl 1):S142-S145

20. Cozzolino DJ, Lipshultz LI (2001) Varicocele as a progressive lesion: positive effect of varicocele repair. Hum Reprod Update 7(1):55-58

21. Abdel-Meguid T (2012) Predictors of sperm recovery and azoospermia relapse in men with nonobstructive azoospermia after varicocele repair. J Urol 187(1):222-226

22. Esteves SC, Glina S (2005) Recovery of spermatogenesis after microsurgical subinguinal varicocele repair in azoospermic men based on testicular histology. Int Braz J Urol 31 (6):541-548

23. Inci K, Hascicek M, Kara O, Dikmen AV, Gürgan T, Ergen A (2009) Sperm retrieval and intracytoplasmic regions sperm injection in men with nonobstructive azoospermia, and treated and untreated varicocele. J Urol 182(4):1500-1505

24. Tiseo BC, Russell PH, Tanrikut C (2015) Surgical management of nonobstructive azoospermia. Asian J Urol 2(2):85-91

25. Pryor JL, Howards SS (1987) Varicocele. Urol Clin North Am 14:499-513

26. Cayan S, Shavakhabov S, Kadioglu A (2009) Treatment of palpable varicocele in infertile men: a meta-analysis to define the best technique. J Androl 30:33-40

27. Palermo G, Joris H, Devroey P, Van Steirteghem AC (1992) Pregnancies after intracytoplasmic injection of single spermatozoon into an oocyte. Lancet 340:17-18

28. Aboulghar MA, Mansour RT, Serour Gl, Fahmy I, Kamal A, Tawab NA Amin YM (1997) Fertilization and pregnancy rates after intracytoplasmic sperm injection using ejaculate semen and surgically retrieved sperm. Fertil Steril 68:108-111 
29. Ishikawa T, Kondo Y, Yamaguchi K, Sakamoto Y, Fujisawa M (2007) Effect of varicocelectomy on patients with unobstructive azoospermia and severe oligospermia. BJU Int 101:216-268

30. Kim ED, Leibman BB, Grinbat DM, Lipshultz LI (1999) Varicocele repair improves semen parameters in azoospermic men with spermatogenic failure. J Urol 162:737-740

31. Aboulghar MA, Mansour RT, Serour GI, Fahmy I, Kamal A, Tawab NA et al (1997) Fertilization and pregnancy rates after intracytoplasmic sperm injection using ejaculate semen and surgically retrieved sperm. Fertil Steril 681:108

32. Szabo R, Kessler R (1984) Hydrocele following internal spermatic vein ligation: a retrospective study and review of the literature. J Urol 132:924

33. Mellinger BC (1995) Varicocelectomy. Tech Urol 1:188-196
34. Amelar RD (2003) Early and late complications of inguinal varicocelectomy. J Urol 170:366-369

35. Schlegel PN, Palermo GD, Goldstein M, Menendez S, Zaninovic N, Veeck $\mathrm{LL}$ et al (1997) Testicular sperm extraction with intracytoplasmic sperm injection for nonobstructive azoospermia. Urology 49:434-440

36. Devroey P, Liu J, Nagy Z, Gossens A, Tournaye H, Camus H et al (1995) Pregnancies after testicular sperm extraction and intracytoplasmic sperm injection in non-obstructive azoospermia. Hum Reprod 10:1457-1460

\section{Publisher's Note}

Springer Nature remains neutral with regard to jurisdictional claims in published maps and institutional affiliations.

\section{Submit your manuscript to a SpringerOpen ${ }^{\circ}$ journal and benefit from:}

- Convenient online submission

- Rigorous peer review

- Open access: articles freely available online

- High visibility within the field

- Retaining the copyright to your article

Submit your next manuscript at $\boldsymbol{\nabla}$ springeropen.com 